\title{
Study on failure mechanism of Cu-polyethylene-Cu sandwich structure by molecular dynamics simulation
}

\author{
Changyu Meng ${ }^{\mathrm{a}, \mathrm{b}}$, Lijuan Liao ${ }^{\mathrm{a}, *}$, Chenguang Huang ${ }^{\mathrm{a}, \mathrm{b}}$

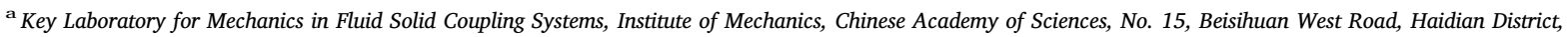 \\ Beijing 100190, China \\ ${ }^{\mathrm{b}}$ School of Engineering Science, University of Chinese Academy of Sciences, No. 19(A), Yuquan Road, Shijingshan District, Beijing 100049, China
}

\section{A R T I C L E I N F O}

\section{Keywords:}

Failure mechanism

Sandwich structure

Molecular dynamics

Thickness-dependence

Micro-void nucleation

Dihedral distribution

\begin{abstract}
A B S T R A C T
The tensile failure mechanism of Cu-Polyethylene (PE)-Cu (CPC) sandwich structure was clarified by molecular dynamics (MD) simulations subjected to a uniaxial tensile loading at microscopic scale. The sensitivity analysis of parameters such as mixing rules in describing the interaction between the wall $(\mathrm{Cu})$ and the sandwich layer (PE), model size, relaxation time for equilibrium and initial velocity distribution was carried out to verify the rationality of modeling. The evolutions of stress-strain relationship and each potential energy component were provided to describe the failure process of the structure. The peak of non-bond energy shows a delay compared to the yield point in stress-strain curve, which coincides with the local maximum point of the trans-fraction curve of dihedral angles. After that, an inflexion appeared in the trans-fraction curve indicates an energy transport process, which corresponds with the slope change of the stress-strain curve. It is assumed that the dihedral distribution plays a crucial role in the damage process of CPC structure. In addition, the temperature field and the density profile were adopted to predict the position of damage initiation, which was confirmed by the microstructure evolution. The intrinsic thickness-dependence of CPC was explored by taking the coupling effect of bridging and entanglement into account, which is in reverse proportion with the yield strength of CPC.
\end{abstract}

\section{Introduction}

Interfaces between polymers and metals are common in industrial systems, such as in adhesive structures for automotive and aircraft applications, microelectronic device packaging and coatings [1]. Generally, an interface forms with adhesion when the physical and chemical reactions occur between two phases (matrix and polymer layers). As an effective component to transfer stress and bear the structural loadings, the bonding structure composed of adhesive and metal matrix is the focus of our research.

The two-phase interface structure formed by the combination of metal matrix and polymer, as a weak link, is most likely to occur damage during the whole structure bearing a large loading. The failure process of the two-phase structure is often different from that of a single phase, which depends not only on the physical properties such as toughness, strength and stiffness of the polymer, but also on the nature of entire structure [2]. In order to make the bonding structure more economical and reliable, it is necessary to deeply understand and clearly describe its mechanical behavior under loading, so as to provide a theoretical basis for optimizing the design related to the bearing capacity of the component.

The general polymer materials have both the characteristics of entanglement and cross-linking [3]. The micro-holes in the polymer layer do not aggregate and generate large cracks when the damage occurs. In contrast, fibrillation occurs due to a certain mechanism in failure process, which is described as macroscopic silver craze [4]. In addition, metal substrates are typically several orders of magnitude stronger than polymers. Three main categories as cohesive failure (failure in the polymer layer), adhesive failure (debonding at the polymer-adherend interface) and mixed failure (combined with cohesive-adhesive failure) are taken into account, irrespective of the failure of the metal matrix $[5]$.

In the early studies, research scholars tended to accumulate and analyze experimental data and tried to establish analytical or numerical models. The load-testing modes for the adhesive system mainly include tensile, shear and split. Accordingly, the relationship between the local separation displacement and the traction stress at the bonding interface can directly express its mechanical response. The experimental data can be integrated into a specific mathematical model to obtain the normal or shear separation strength [6,7]. Liao et al. [8] carried out the

\footnotetext{
* Corresponding author.

E-mail addresses: mengchangyu@imech.ac.cn (C. Meng), liaohuanxin@hotmail.com, lj1@imech.ac.cn (L. Liao), huangcg@imech.ac.cn (C. Huang).
} 
experimental and finite element (FE) calculation for single-lap adhesive joints, which presented the different rupture initiation positions under impact and static tensile loadings. As one of the important parameters during the bonding process, the thickness of the adhesive layer, especially with a small value, has a great influence on the interfacial strength. When the thickness exceeds a certain critical value [9], the area near the wall will not be affected by plastic deformation [10]. In addition, the design and fabrication of fiber-reinforced composite structures [11-13] can also give us the inspiration about the design of adhesive joints for improving their properties under mixed loadings. As for the analytic models, the descriptions of the two-dimensional stress distribution in interfacial structures at the continuum level are favored largely. Most of existing analytical models focus on linear elastic solutions of polymers and matrix under different external loadings [14,15]. The difficulty for obtaining the analytic solution increases with the uprising complication of the forms used to complete the analytic model. Therefore, researchers usually simplify actual problems as the idealized ones to fit for these developed analytical solutions. In the aspect of numerical models, the cohesive zone model (CZM) can characterize the elastic-plastic behavior of the adhesive structure [16] to predict the overall failure of adhesive bond, which can be easily implemented by FE calculation. Liao et al. [17] studied the load-bearing capacity and the damage level of the double scarf joint (DSJ) by FE method using the mixed-mode CZM with a bilinear shape. However, the cohesive parameters for the same loading method of different materials or the different loading methods of the same material are very different. Correspondingly, many researchers contributed to study the influence of cohesive parameters on the prediction accuracy of the overall mechanics behavior of the adhesive structures [18-20].

Considerable results were obtained aiming to the behaviors of interfacial structures at the macro scale. However, whether in experimental or theoretical studies, the existing characterizations of interface damage mainly focused on macroscopic continuum mechanics and phenomenological hypothesis. For lacking of detailed description of the objective physical image, it is necessary to seek the intrinsic mechanism of the phenomenon of the whole damage process.

Molecular dynamics (MD) simulation can be chosen to study the failure behavior of interfacial structures at atomic scale. Alder and Wainwright [21] adopted the hard-sphere model to study the equation of state for gases and liquids. As development of the basic theories such as computing method and force field, MD method has been gradually applied to the fields of structure and engineering. Some investigators discussed the relationship between structural failure and temperature, interfacial interaction, loading rate by MD [22], whose results agree well with experimental data [4]. Therefore, MD method is an ideal solution to capture the mechanical behavior of the adhesive structures with extreme small thicknesses. In addition, this method can also be adopted to simulate the response of the structures in extreme environments like impact loadings, thermal circumstances, etc. It provides the possibilities of clearly understanding the behavior of polymers at microscopic scale, and grasping the relationship between the chemical structure and physical properties of polymers. Fan et al. [23] obtained the traction-separation relationship of the epoxy/copper interfacial structure using MD method. The constitutive relation was applied to FE analysis in CZM model. Zhou et al. [24] employed this strategy to calculate the cohesive parameters of brittle materials in mixed mode. These studies provide an idea to examine the multi-scale coupling of polymer/metal interface.

The failure mode of an interfacial system containing organic and inorganic materials usually depends on several aspects [9], such as the properties of the material itself (lattice parameters, glass transition temperature, etc.), the constraint effect (adhesive size, interfacial adhesion area, the degree of bonding uniformity, etc.) as well as the external conditions (loading rates, temperature, etc.). The failure of an interfacial system is essentially the result of multi-factor coupling. In previous studies, we analyzed the coupling effect of adhesive type and geometry (adhesive thickness and scarf angle) on the mixed-mode failure of DSJ using dimensional analysis and FE method (CZM) $[25,26]$. However, the intrinsic mechanism of these influential parameters is still unclear owing to the limitation of the scale. Explorations in smaller scale like atomic- or molecular-scale should be carried out to provide multiscale understandings. There were several investigations on the mechanical responses of a sandwich structure (adhesive-like) consisting of amorphous polyethylene (PE) chains and face-centered cubic crystal copper layers (Cu-PE-Cu, CPC) in the framework of MD, and the branching and cross-linking of the polymer layer were out of consideration for simplicity. However, there were still some problems. The overall sizes in simulations are too small to represent the characteristics of real systems [5]. The description of the relationship between PE interface size and overall strength is too brief [9] to reach a quantitative understanding on the failure mechanism. Moreover, an intuitive description of the failure process of the interfacial system is eagerly to be developed.

The paper is organized as follows. In Section 2, the modeling procedures of bulk amorphous PE and CPC sandwich are described. In Section 3, the damage evolution until failure of CPC sandwich is simulated. The corresponding methods of characterization are also provided. More importantly, the thickness effects coupling with entanglement and bridging are discussed.

\section{Simulation method}

\subsection{Force field}

Taking efficiency and accuracy into account as much as possible, a $-\mathrm{CH}_{2}$ - monomer (and the terminal $\mathrm{CH}_{3}-$ monomer) in a $\mathrm{PE}$ chain was treated as a united atom (UA) [27,28]. Copper layers were considered as rigid bodies without interactions between inner atoms. Accordingly, the atoms in copper layer move as a single group. At the interface of two materials, we assume that there is only the non-bond interaction between copper atoms and PE UA. All MD simulations were implemented by LAMMPS coding [29], and all the visualization operations were performed by OVITO [30].

Dreiding potential [31] was selected to characterize the intra- and inter-molecular interactions of the PE chains, which are with four terms, namely bond stretching (with a subscript of bond), bond angle changing (with a subscript of angle), dihedral angle rotation (with a subscript of dihedral) and intermolecular interactions (with a subscript of non-bond). The total potential energy (with a subscript of total) and each component are expressed by Eqs. (1)-(5), respectively.

$E_{\text {total }}=E_{\text {bond }}(r)+E_{\text {angle }}(\theta)+E_{\text {dihedral }}(\phi)+E_{\text {non-bond }}(r)$

$E_{\text {bond }}=\frac{1}{2} K_{b}\left(r-r_{0}\right)^{2}$

$E_{\text {angle }}=\frac{1}{2} K_{\theta}\left(\theta-\theta_{0}\right)^{2}$

$E_{\text {dihedral }}(\phi)=\sum_{i=0}^{3} C_{i}(\cos \phi)^{i}$

$E_{\text {non-bond }}(r)=4 \varepsilon\left[\left(\frac{\sigma}{r}\right)^{12}-\left(\frac{\sigma}{r}\right)^{6}\right], \quad r \leqslant r_{c}$

where $K_{\mathrm{b}}$ and $K_{\theta}$ are the stiffness constants of the bond length and the bond angle, respectively. $r_{0}$ and $\theta_{0}$ are the equilibrium bond length and the bond angle, respectively. $C_{i}(i=1,2,3)$ represents the coefficients in Eq. (4) to describe the dihedral angle.

Intermolecular potential energy, as shown in Eq. (5), describes the interactions among the PE chains themselves and that between the copper atoms and the PE UA. Expressed in terms of Lennard-Jones (LJ) $12-6, \sigma$ in Eq. (5) defines the equilibrium distance between atoms as the energy is minimum for $r=2^{1 / 6} \sigma$, and $\varepsilon$ represents the depth of the 

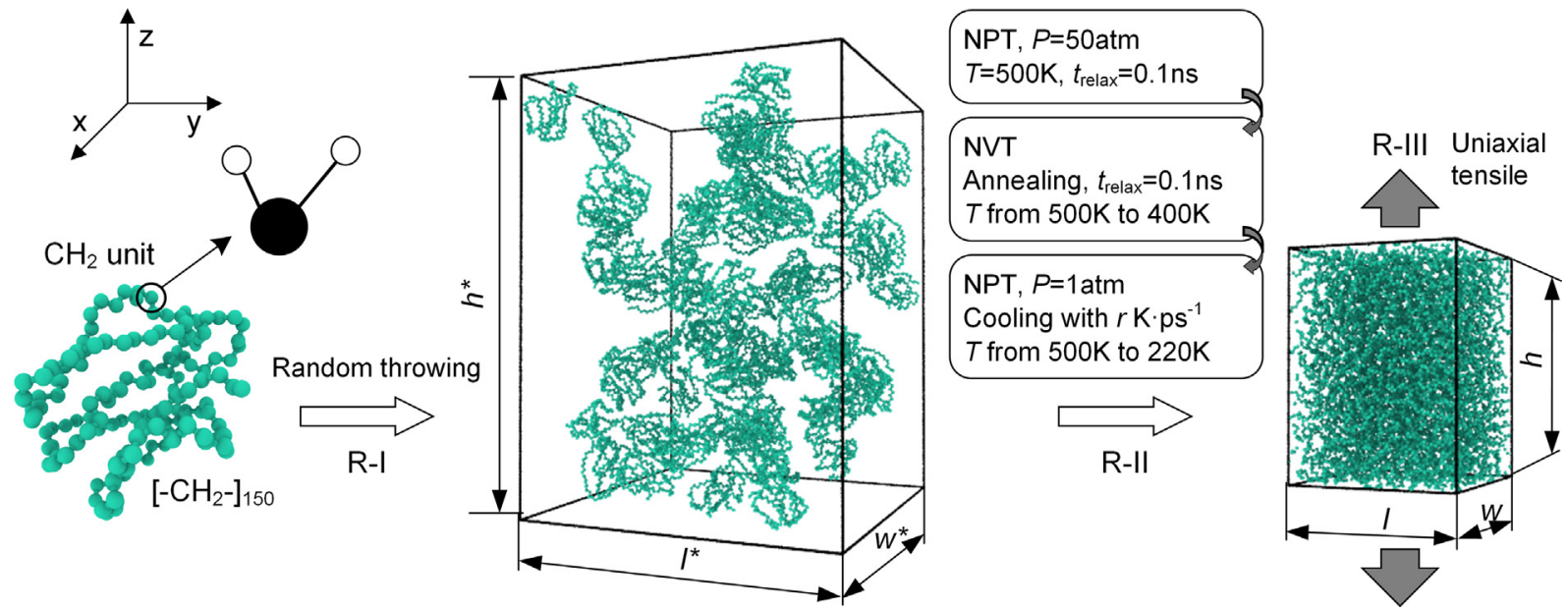

Fig. 1. Modeling process of an amorphous block PE system.

potential well. The potential parameters used to describe PE could refer to Hossain et al. [32]. The potential parameters characterizing copper are from Heinz et al. [33], which are only used to describe the interfacial interaction between copper atoms and PE UA with the arithmetic (Lorentz-Berthelot, LB) mixing rule [5] (with a subscript of cp, as expressed in Eq. (6), the detailed sensitivity analyses on mixing rules are discussed in Sections 2.4 and 3.1). The LJ potential cut-off radius for all non-bond interactions was $10 \AA\left(10^{-9} \mathrm{~m}\right)$.

$\varepsilon_{\mathrm{cp}}=\sqrt{\varepsilon_{\mathrm{Cu}} \varepsilon_{\mathrm{PE}}}, \sigma_{\mathrm{cp}}=\frac{\sigma_{\mathrm{Cu}}+\sigma_{\mathrm{PE}}}{2}$

\subsection{Modeling and tensile simulations of amorphous PE}

Before simulating confined CPC sandwich structures, the suitability of the UA potential needs to be verified by reproducing the physical properties of PE. In addition, the reliability of the equilibrium process and the appropriate size of the middle PE layer for CPC structures should be determined in advance.

Fig. 1 shows the modeling process of an amorphous block PE system. Three-direction periodic boundary condition was defined with a time step of $\Delta t=1 \mathrm{fs}$. Han et al. [34] pointed out that a single PE chain with less than one hundred repeat units in the simulation model may result in un-real physical responses. Accordingly, a long straight PE chain with one-hundred-fifty units was created followed by a relaxation procedure to obtain a curled PE strand. Then the PE chains were thrown randomly into a box with a size of $l^{*} \times w^{*} \times h^{*}$ by Packmol [35] during R-I process. After a series of relaxation steps under different ensembles during R-II, the initial system with small density $\rho^{*}\left(\sim 0.1 \mathrm{~g} \cdot \mathrm{cm}^{-3}\right)$ was converted into one with a real density $\rho\left(\sim 0.9 \mathrm{~g} \cdot \mathrm{cm}^{-3}\right)$ at $T=220 \mathrm{~K}$. The final configuration at equilibrium was adopted to obtain the glass transition temperature $\left(T_{\mathrm{g}}\right)$ and the mechanical behaviors subjected to tensile loadings.

$T_{g}$ of amorphous block PE system was calculated according to temperature-dependence of density. The PE system at equilibrium was firstly subjected to an annealing process between $500 \mathrm{~K}$ and $400 \mathrm{~K}$ in NVT ensemble. Then it was cooled down to $20 \mathrm{~K}$ with a cooling rate of $r=0.8 \mathrm{~K} \cdot \mathrm{ps}^{-1}$ in NPT ensemble. Three systems (Si, $\left.i=1,2,3\right)$ with different initial sizes were established and then equilibrated by the procedure described above. The sizes at equilibrium and glass transition temperatures of these amorphous PE systems are shown in Table 1. Each physical quantity listed in Table 1 was averaged by three samples to carry out a statistical analysis. It can be observed that $T_{\mathrm{g}}$ of different systems are around $250 \mathrm{~K}$, which are within the experimental range from 190 to $300 \mathrm{~K}$ [36].

The mechanical responses of the amorphous PE systems under
Table 1

Size and properties of amorphous PE systems at equilibrium.

\begin{tabular}{lllll}
\hline No. & $l(w)(\AA)$ & $h(\AA)$ & $\rho\left(g \cdot \mathrm{cm}^{-3}\right)$ & $T_{\mathrm{g}}(\mathrm{K})$ \\
\hline S1 & $40.29 \pm 0.04$ & $80.58 \pm 0.07$ & $0.884 \pm 0.000$ & $252.3 \pm 2.5$ \\
S2 & $60.39 \pm 0.04$ & $80.52 \pm 0.05$ & $0.881 \pm 0.001$ & $257.8 \pm 2.5$ \\
S3 & $80.51 \pm 0.06$ & $80.51 \pm 0.06$ & $0.883 \pm 0.001$ & $254.5 \pm 1.7$ \\
\hline
\end{tabular}

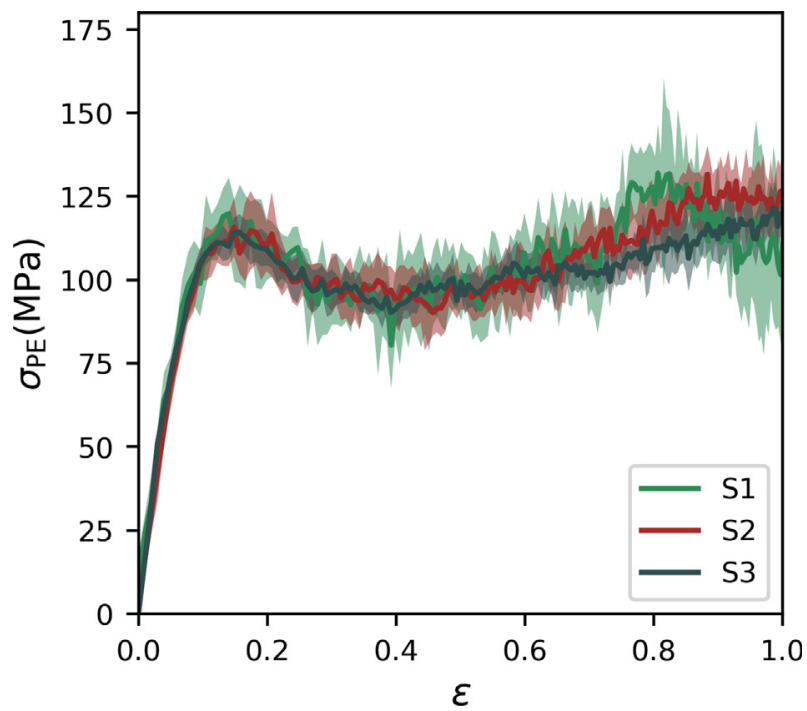

Fig. 2. Stress-strain response of three PE systems under uniaxial tension with $\dot{\varepsilon_{z}}=10^{10} \mathrm{~s}^{-1}$ and $T=220 \mathrm{~K}$.

tensile loadings were also discussed using different initial configurations as mentioned above. The tensile strain was applied once per 2000 time steps [32] and the virial stress [5] was calculated $\left(\dot{\varepsilon}_{z}=10^{10} \mathrm{~s}^{-1}\right.$, $P_{x}=P_{y}=0 \mathrm{~atm}, T=220 \mathrm{~K}, \Delta t=1 \mathrm{fs}$ ). As shown in Fig. $2, \sigma_{\mathrm{PE}}$ and $\varepsilon$ are the stress and strain of the given PE system, respectively. The solid line is the average stress in $z$ direction of each system (Si, $i=1,2,3)$ at different strains, and each stress value adopted was the average of them in last 1000 time steps. The error band is demonstrated by translucent area correspondingly. Four classical stages either in MD simulations [32] and experiments [37]: elastic, yield, softening and hardening can be observed in Fig. 2 .

It can be assumed that the modeling and relaxation of amorphous PE systems adopted in the present study are reasonable according to the verifications of $T_{\mathrm{g}}$ and the stress-strain curves. Even though the $T_{\mathrm{g}}$ of different PE systems are consistent with each other, a larger oscillation 


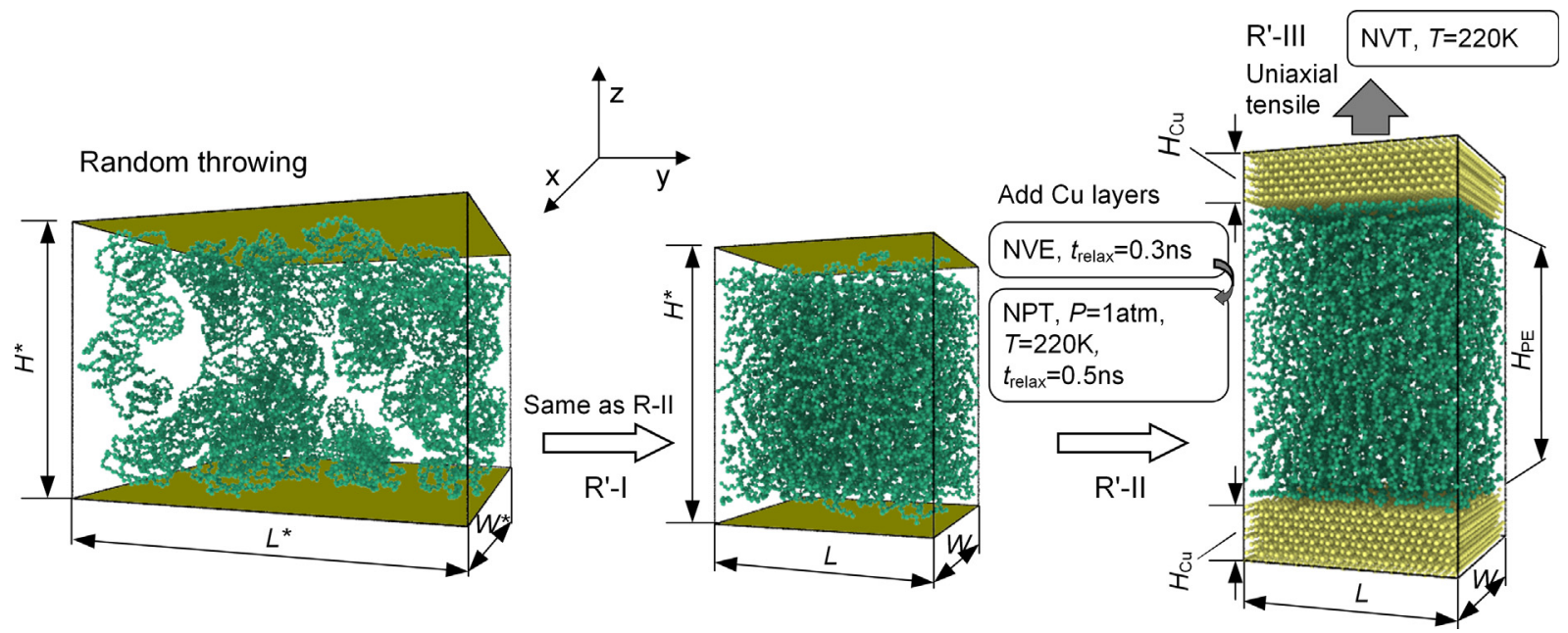

Fig. 3. Modeling process of the CPC sandwich.

amplitude was observed in the stress-strain curve of S1 than that in other two systems. A more reasonable statistical average in larger size system, like S2 and S3, can be obtained. Taking the balance between efficiency and accuracy into account, the lateral size of S2 ( $l=w \cong 60 \AA$ ) was chosen as the target value for the lateral dimensions of equilibrated sandwich structures in the following section.

\subsection{Modeling process of the CPC sandwich}

The modeling process of CPC sandwich is shown in Fig. 3, which refers to the method of building the PE layer as shown in Fig. 1. It should be noticed that before adding two copper layers to the top and bottom of PE layer, the upper and lower walls of PE box in $z$ direction was fixed and they possess the same non-bond interaction as the LJ interaction between the copper atoms and the PE UA, as shown in Eq. (5). The height of PE layer was fixed as $H^{*}$. The height of PE layer $H_{\mathrm{PE}}$ at equilibrium is close to $H^{*}$. After two copper layers were placed on the top and bottom of the PE layer, the whole system needs a sufficient time of relaxation under the NVE and NVT ensembles to eliminate the stress oscillations in the $z$ direction.

In the initial processes of modeling, the height $H^{*}$ of PE layer was set as $80 \AA$ [9] (as shown in Fig. 3). The close packed plane (1 1 1) of the copper was selected in generating the substrates of CPC sandwich. After relaxation described in Fig. 3, the height of PE layer $H_{\mathrm{PE}}$ decreases slightly to $79.94 \pm 2.07 \AA$ owing to the wall effect. In addition, the lateral dimensions of the equilibrated CPC systems are about $60 \AA$ ( $L=W \cong 60 \AA$ ), which are similar to those of the S2 system described in Section 2.2. The tensile deformation of CPC sandwich was simulated by moving the upper copper layer with a rate of $\beta=1 \AA \cdot \mathrm{ps}^{-1}$. The temperature was kept at $220 \mathrm{~K}$, which is below $T_{g}$ to guarantee the glassy state of PE.

\subsection{Sensitivity analyses of simulation parameters for the CPC sandwich}

The effects of several independent parameters, such as mixing rules used in describing the interactions between copper atoms and PE UA, cooling rate $r$ during relaxation ( $\mathrm{R}^{\prime}-\mathrm{I}$ in Fig. 3), relaxation time $t_{\text {relax }}\left(\mathrm{R}^{\prime}-\right.$ II in Fig. 3) and random velocity distribution method for temperature initiation, on the stress-strain relationship of CPC sandwich were also examined. The nominal stress $\sigma_{\mathrm{n}}$ that refers to the average of the virial stress in $\mathrm{z}$ direction of all PE united atoms was calculated in consideration of the inhomogeneity of the stress field in the PE layer during the loading process. As shown in Fig. 4, the solid line is the average nominal stress at different strains of six separate simulation runs with different random velocity seeds. The error bands, which are the translucent areas around the solid line, represent the standard deviations of these different simulations.

Mixing rules might affect the mechanical responses of CPC structures for they were shown to have an influence on the accurate values of thermodynamic quantities like density for multi-type atoms mixtures [38]. However, for our main focus is the microstructure evolution of the system, the adoption of mixing rules would not influence the semiquantitative results greatly (as shown in Fig. 4(a)). It can be explained by the small difference on potential parameters (as Table S1 and Fig. S1 in supplemental material) and at the low loading rates (as the $\beta$ $=1 \AA \cdot \mathrm{ps}^{-1}$ case in Figs. S2 (a) and S3 in supplemental material). In addition to mixing rules, the effects of the other three parameters discussed here are also negligible as shown in Fig. 4(b)-(d). Furthermore, it also shows that the modeling method described above is acceptable. Correspondingly, the LB mixing rule in describing the molecular interactions between $\mathrm{Cu}$ and $\mathrm{PE}$, the cooling rate $r$ as $0.8 \mathrm{~K} \cdot \mathrm{ps}^{-1}$, the relaxation time $t_{\text {relax }}$ as $0.5 \mathrm{~ns}$ and the uniform velocity distribution for temperature initiation were chosen in the present simulation.

\section{Results and discussion}

\subsection{Failure process of CPC sandwich structure}

The physical responses of CPC sandwich subjected to tensile load are illustrated in Fig. 5. The evolution of microstructures from nucleation to complete failure with partial enlargements at five marked points ( $\mathrm{a} \sim \mathrm{e}, \varepsilon=0,0.1,0.2,0.4$ and 1.2) at given strains as well as the stress-strain curve are plotted in Fig. 5(a). The solid lines and corresponding semi-transparent areas demonstrate the average values and error bands using statistical analysis, respectively. Across yield point $\mathrm{b}$ ( $\varepsilon=0.1$ ), the aggregation and expansion of the micro-pores after $c$ point $(\varepsilon=0.2)$ result in the obvious voids in d point $(\varepsilon=0.4)$ in the middle area of PE layer. The straighten chains, which bear loadings, are observed clearly in the drawing of partial enlargement. When the strain reaches 1.2 , the structure is completely broken down without stresses.

Each component of potential energy evolves gradually with the stress state evolution of the sandwich structure as $\varepsilon$ increases. Fig. 5(b) shows the evolution of the mean value of each component of potential energy for six samples at the given strain $\varepsilon$. The ordinate $\Delta E$ is the difference between the energy of the structure at a particular strain state and the energy at the initial moment $(\varepsilon=0)$. As illustrated in Fig. 5(b), the contribution of non-bond energy $E_{\text {non-bond }}$ to the total potential energy $E_{\text {total }}$ is the largest. $E_{\text {non-bond }}$ increases linearly up to a peak (the strain at this point is marked as $\varepsilon_{\mathrm{E}}$ ), which is after the yield point of the stress-strain curve (in Fig. 5(a)). In addition, the bond energy $E_{\text {bond }}$ rises gradually resulting from bond stretching during 

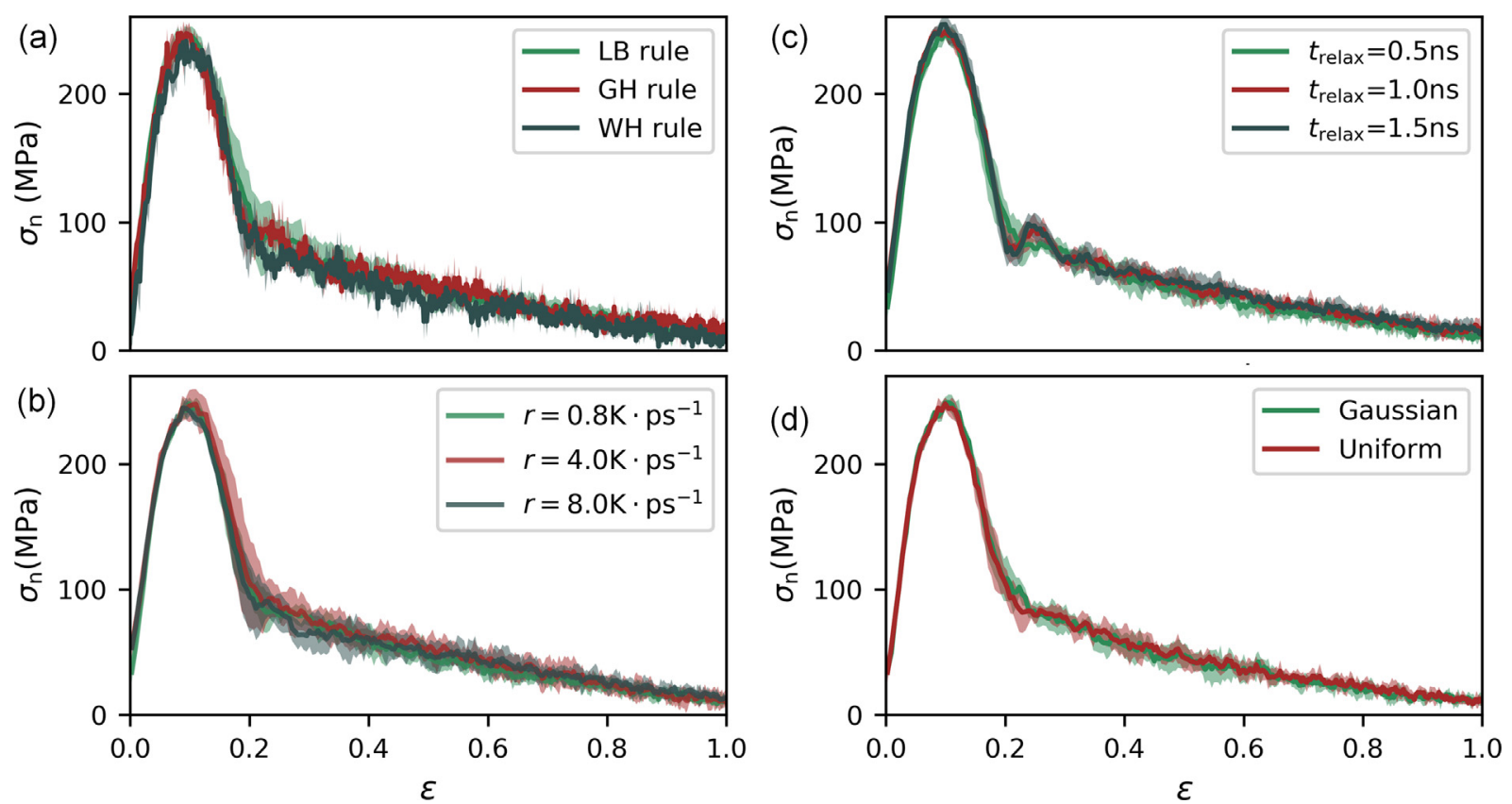

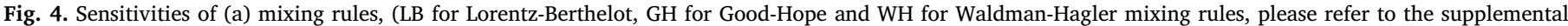

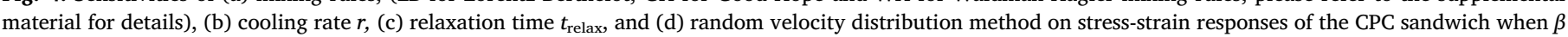
$=1 \AA$ A $\mathrm{ps}^{-1}$.

elastic deformation. The micro-pores are assumed to appear at a certain strain between 0.1 and 0.2 (in the range of makers b and c), which can be concluded from the drawings of partial enlargement as shown in Fig. 5(a). The phenomenon that visible voids form and propagate with a delay across yield point $\mathrm{b}$ results in the latter decline of $E_{\text {non-bond. }}$ In addition, the similar trends of $E_{\text {dihedral }}$ and $E_{\text {angle }}$ can be found in Fig. 5(b). $E_{\text {dihedral }}$ experiences an initial slow decline followed by a plateau region. An inflection near the strain of $\varepsilon_{\mathrm{E}}$ brings a fair climb and a moderate drop. Hossain et al. [32] argued that the distribution of the dihedral angles during the stretching of PE is more notable than that of bond angle. Accordingly, we will focus more on the distribution and energy of the dihedral angles in the sandwich systems to explore the intrinsic mechanism of failure.

The dihedral component of potential energy is lowest when the dihedral angles distributes around $0^{\circ}$ or $180^{\circ}$ [9], which is called the trans state (there is also a gauche state around $120^{\circ}$ ) [3]. The ratio of the number of dihedral angles from $160^{\circ}$ to $180^{\circ}$ to the number of total dihedral angles was defined as trans fractions $\chi$, which indicates the relative number of low-energy dihedral structures. The larger value of $\chi$ represents the higher load-bearing capacity. The evolution of transfraction $\chi$, which is shown in Fig. 5(c), comes from the mean value of six samples at the given strain $\varepsilon$. As shown in Fig. 5(c), the evolution of $\chi$ experiences an initial rise to the first peak when the strain reaches $\varepsilon_{\mathrm{E}}$, as shown in Fig. 5(b). It indicates that the PE layer is extremely unstable at this point, which induces the appearance of micro-pores. In addition, a kick point marked as c corresponds to the rise stage of dihedral energy $E_{\text {dihedral }}$ in Fig. 5(b) reflects an energy transport resulting in the slope change of the stress-strain curve in Fig. 5(a). In addition, the overall trend of variation of $\chi$ is increasing as $\varepsilon$ rises, which reveals that the number of the lower energy dihedral angles increases gradually resulting in the plastic deformation of the structure. The gradual decrease of dihedral energy leads to the complete failure of the CPC sandwich structure.

Fig. 6 illustrates the variation of PE density in $z$ direction (solid curve), the average kinetic energy $\overline{\mathcal{K}}$ (contour) and the evolution of microstructure in PE layer of the CPC sandwich at given strains (corresponding to the three makers $\mathrm{a}, \mathrm{b}$ and $\mathrm{c}$ in Fig. 5, respectively) when loading rate $\beta$ is $1.0 \AA \cdot \mathrm{ps}^{-1}$ (left column) and $5.0 \AA \cdot \mathrm{ps}^{-1}$ (right column), respectively. These distributions were discussed by the projection in the $y z$ plane. It can be found that the wall-effect brings higher density at the vicinity of the wall. In addition, it is necessary to point out that the distribution of the density in $z$ direction is oscillatory, which is consistent with the distribution probability of particles with finite size in non-periodic line in statistical mechanics [39]. It supports that the current calculations meets statistical mechanics requirements. As described in Fig. 6, the nucleation of micro-pores at $\varepsilon=0.2$ results in the falling of local density in the range of $0.34<\hat{H}<0.52$ when $\beta=1.0 \AA \cdot \mathrm{ps}^{-1}$ and $0.72<\hat{H}<0.88$ when $\beta=5.0 \AA \cdot \mathrm{ps}^{-1}$, respectively, where $\hat{H}$ is the normalized position in z-direction. The trough region illustrated in the density profile represents the location of damage.

The initiation of damage requires energy absorption to propagate progressively, which brings high temperature. The temperature distribution of PE layer can be calculated according to the average kinetic energy $\bar{\kappa}$ by the expression $\bar{\kappa}=3 / 2 k_{\mathrm{B}} T$, where $T$ and $k_{\mathrm{B}}$ are the absolute temperature and the Boltzmann constant, respectively. As shown in Fig. 6, the high temperature regions with low density near the upper wall can be observed obviously when $\beta=5.0 \AA \cdot \mathrm{ps}^{-1}$. On the contrary, the lower moving rate $\left(\beta=1.0 \AA \mathrm{ps}^{-1}\right)$ leads to the occurrence of high temperature and low density in the middle of PE layer. As a direct reflection of the atomic velocity, the temperature field could be adopted to predict the position of void nucleation effectively.

It is necessary to point out the sensitivity of mixing rules in describing the interaction between the wall and the sandwich layer at high loading rate as $\beta=5.0 \AA \mathrm{ps}^{-1}$. As shown in Fig. S2(b) in supplemental material, the stress-strain curves appear slightly different at large strain $(\varepsilon>0.5)$. However, the density profile and the average kinetic energy distribution as shown in Fig. 6 were mainly in the initial deforming process $(\varepsilon<0.2)$. Accordingly, little influence has variation of the mixing rules on the results.

\subsection{Thickness-effect on the yield strength}

Adnan and Sun [5] and Kulmi and Basu [9] have analyzed the thickness-effect of middle layer on the yield strength of sandwich structures with polymers. However, neither of them clearly explained 

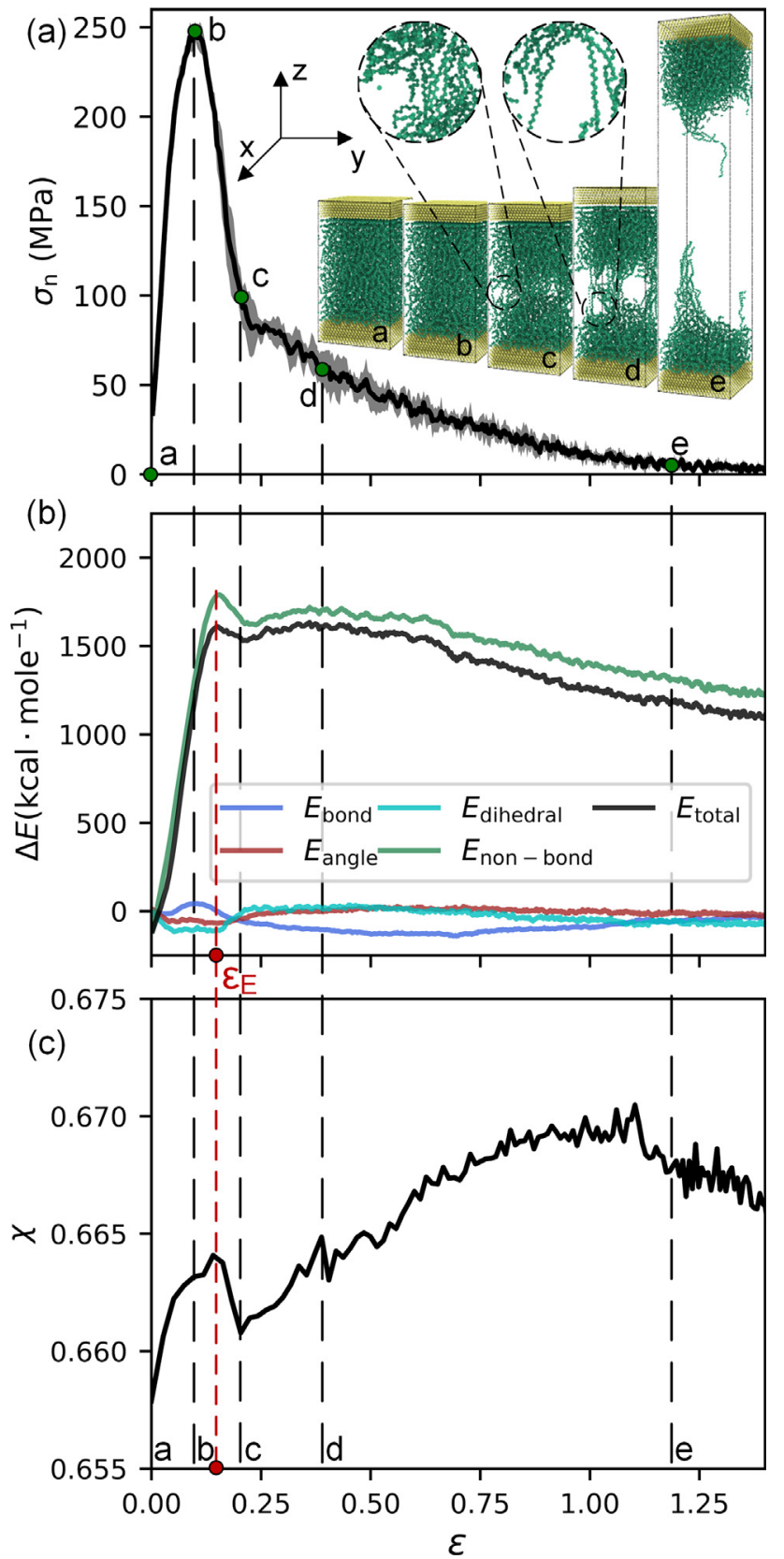

Fig. 5. Evolutions of (a) stress-strain curve, (b) each component of potential energy and (c) trans-fraction $\chi$ of CPC sandwich during tensile load process.

the specific mechanism from the microscopic point of view. The complex network structure is the vital factor for the mechanical behavior of polymers [3]. Without taking cross-linking into account, the effects of entanglement and bridging of PE chains were discussed in this study. Bridging is a physical effect in the sandwich structure with a thin film, refers to several long PE chains acting as bridges across some fragile regions to prevent voids propagation. It should be examined for the sandwich structure during the tensile load.

A series of CPC sandwiches with different $H_{\mathrm{PE}}$ were generated using the method described in Section 2.3. The normalized thickness $H_{\mathrm{n}}=H_{\mathrm{PE}} /\left\langle R_{\mathrm{ee}}\right\rangle$ was introduced, in which $\left\langle R_{\mathrm{ee}}\right\rangle$ is the mean square endto-end distance [3] to represent the size of random linear chains. The characteristic dimensions of six CPC sandwiches with different thickness of PE layer, which are denoted as ISi $(i=1,2,3,4,5$ and 6), are listed in Table 2. It should be noted that the lateral dimension of each sandwich structure is kept at around $60 \AA$.
Inter-chain Contact Fraction (ICF) was selected to characterize the degree of entanglement, which is a structural parameter describing the degree of penetration of PE chains [40]. As shown in Eq. (7), ICF is the ratio of the number of atoms in other chains $\left(N_{\text {inter }}\right)$ near the first neighbor (defined as $r_{1}$, which is decided in radial distribution function) of an atom to the total number of atoms of it $\left(N_{\text {total }}\right)$. Greater value of ICF indicates the greater degree of entanglement and vice versa. The first neighbor $r_{1}$ was determined by the radial distribution function (RDF) of the PE system as shown in Fig. 7. According to Eq. (5), the equilibrium distance of non-bond potential energy is about $2^{1 /}$ ${ }^{6} \sigma \approx 4.5 \AA$. The first neighbor should be the peak in RDF curve after $4.5 \AA$, which is approximately $5 \AA$ as illustrated in Fig. 7. This value is the same as the result obtained by Yu et al. [40]. The variation of ICF with respect to $H_{\mathrm{n}}$ in the range of $r_{1}$ from 4.8 to $5.2 \AA$ is summarized in Fig. 10(b).

$\mathrm{ICF}=\frac{\sum N_{\text {inter }}\left(r_{1}\right)}{\sum N_{\text {total }}\left(r_{1}\right)}$

As for the bridging effect, it refers to the phenomenon that long chains in the middle PE layer restrict the damage propagation, which acts as the bridges to transmit loadings. These bridging chains are not only with high stresses, they also appear different structural features comparing with others. The CPC systems (ISi, $i=1,2, \ldots, 6$ ) were stretched to the post-yielding stage with obvious voids in sequence as shown in Fig. 8. The two schematic diagrams at the upper-left corner in Fig. 8 show the void-nucleation position (V0 point, with a red cross) at the yield strain $\varepsilon_{\mathrm{y}}$ as well as the obvious void (the upper and lower bound points of the main cavity are V1 and V2) at the strain $\varepsilon_{\mathrm{y}}+\Delta \varepsilon(\Delta \varepsilon$ is large enough). We assume that the regions locate outside of the voidnucleation point experience subtle change as quasi static regions. The total size of the quasi-static regions in z direction is $d$, and each $d$ is very close to the $H_{\mathrm{PE}}$ of the corresponding system at equilibrium listed in Table 2. As the precise position of void-nucleation is hard to obtain, V2 point was adopted to replace V0 as a characteristic quantity for determining the bridging chains as shown in Fig. 8. Assumed to prevent damage from initiation and propagation, these bridging chains are most likely located near V0 where initial void appears. Furthermore, playing the role of transferring loadings, the geometry requirement of a bridging chain with a certain length along loading direction should be satisfied. According to the above two assumptions, a sample chain was demonstrated at upper-left schematic diagrams in Fig. 8 of which the location of maximum and minimum value in $z$-coordinate is $\mathrm{z}_{\mathrm{C} 1}$ and $\mathrm{z}_{\mathrm{C} 2}$, respectively, and the three geometry features that bridging chains must have are $\mathrm{z}_{\mathrm{C} 2}<\mathrm{z}_{\mathrm{V} 0}, \mathrm{z}_{\mathrm{C} 1}>\mathrm{z}_{\mathrm{V} 0}$ and $\mathrm{z}_{\mathrm{C} 1}-\mathrm{z}_{\mathrm{C} 2} \geq H_{\mathrm{PE}}^{0} / 2$.

The sum of nominal atomic-stress in the $z$-direction $\left(\sigma_{\mathrm{dz}}\right)$ was calculated for all the united atoms in each PE chain at the yield strain. Here those with top $10 \%$ sum of $\sigma_{\mathrm{dz}}$ were defined as the high-stress PE chains. They are shown as the non-transparent chains (including red and green) in Fig. 9, and the transparent green chains are the rest ones. Bridging effect largely depends on the ratio of bridging chains to the total high-stress ones with large $\sigma_{\mathrm{dz}}$, which contribute greatly to the strength of the system. Correspondingly, bridging chains were selected as high-stress ones qualified the geometry criteria mentioned above. As illustrated in Fig. 9, the chains with high stress are evenly interspersed throughout the PE middle layer. Among them, the number of bridging chains (denoted as red ones) increases with the decreasing thickness of PE layer. In addition, it can be even clearly observed that the bridging chains are in contact with the upper and lower walls in IS1 as showin in Fig. 9, which shows the most significant bridging effect. A parameter $I_{\mathrm{b}}$ was introduced to characterize the bridging effect as expressed in Eq. (8). $N_{\mathrm{s}}$ is the number of high-stress chains in the PE layer. The number of bridging chains is denoted as $N_{\mathrm{b}}$. The bridging parameter $I_{\mathrm{b}}$ is positive correlated with bridging effect. As plotted in Fig. 10(c), it can be seen that $I_{\mathrm{b}}$ inversely related to $H_{\mathrm{n}}$.

$I_{\mathrm{b}}=N_{\mathrm{b}} / N_{\mathrm{s}}$ 

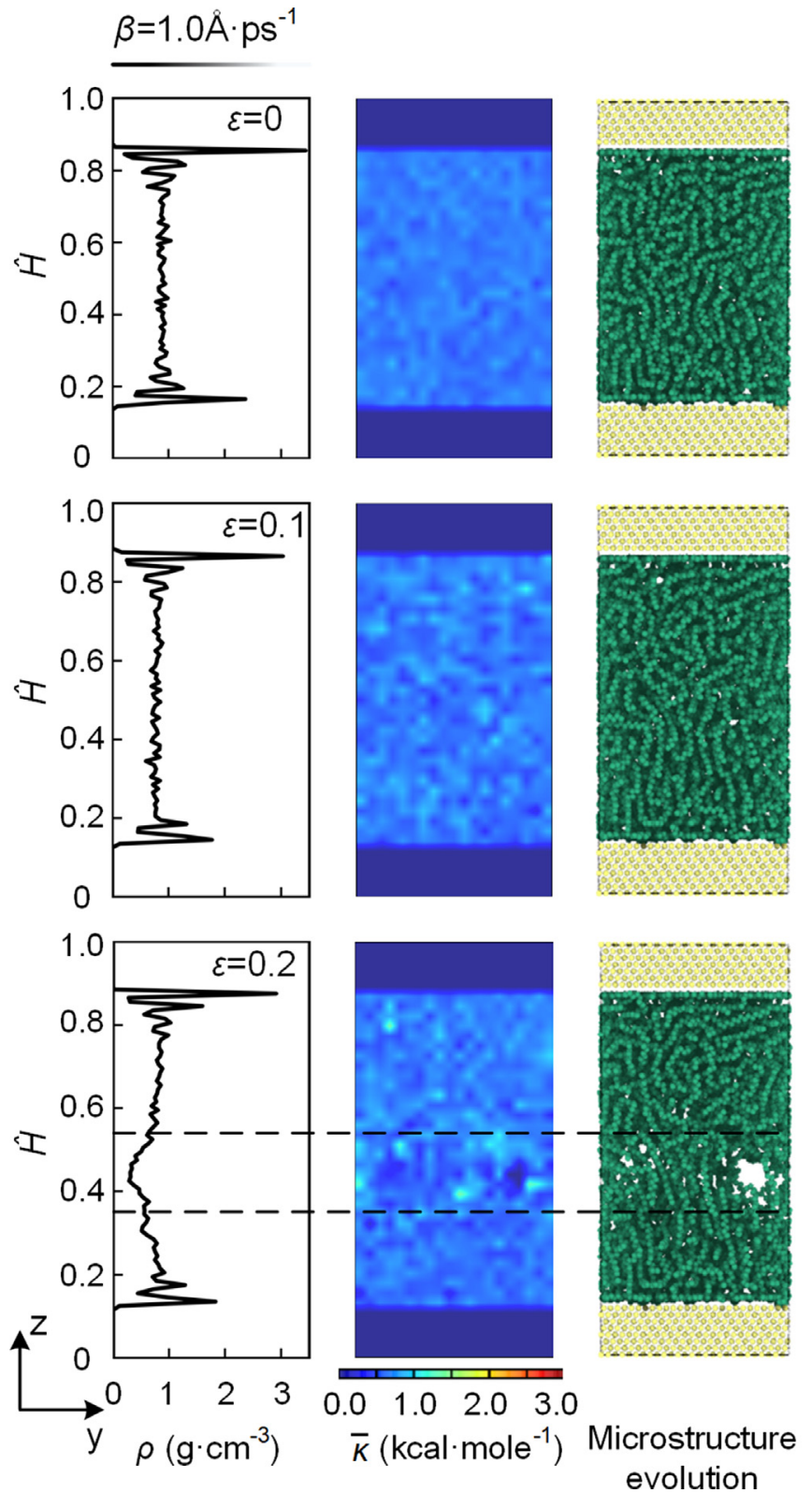

Fig. 6. Density profile, average kinetic energy distribution and microstructure evolution of CPC sandwich at given strains.

Table 2

Characteristic dimensions of different CPC sandwiches at $\varepsilon=0$.

\begin{tabular}{llll}
\hline No. & $<R_{\mathrm{ee}}>(\AA)$ & $H_{\mathrm{PE}}(\AA)$ & $H_{\mathrm{n}}$ \\
\hline IS1 & $30.67 \pm 1.70$ & $40.80 \pm 1.29$ & $1.33 \pm 0.08$ \\
IS2 & $34.16 \pm 0.98$ & $60.58 \pm 1.53$ & $1.77 \pm 0.03$ \\
IS3 & $32.90 \pm 1.15$ & $79.94 \pm 2.07$ & $2.43 \pm 0.11$ \\
IS4 & $32.68 \pm 1.51$ & $98.66 \pm 2.57$ & $3.03 \pm 0.18$ \\
IS5 & $34.85 \pm 0.71$ & $117.13 \pm 3.97$ & $3.36 \pm 0.07$ \\
IS6 & $34.41 \pm 1.17$ & $138.21 \pm 2.70$ & $4.02 \pm 0.18$ \\
\hline
\end{tabular}

The relationship between the yield strength $\sigma_{y}$ of the whole CPC structure and the normalized thickness $H_{\mathrm{n}}$ of each system is given in Fig. 10(a), which is similar to the result presented by Kulmi and Basu [9]. They proposed that the thickness probably has a dual effect on the interface strength. The bridging effect dominates in PE layer with smaller thickness to strengthen the structure. On the other hand, the degree of entanglement is reduced by the high restricting effect owing to the small thickness, which decreases the strength of the sandwich. As
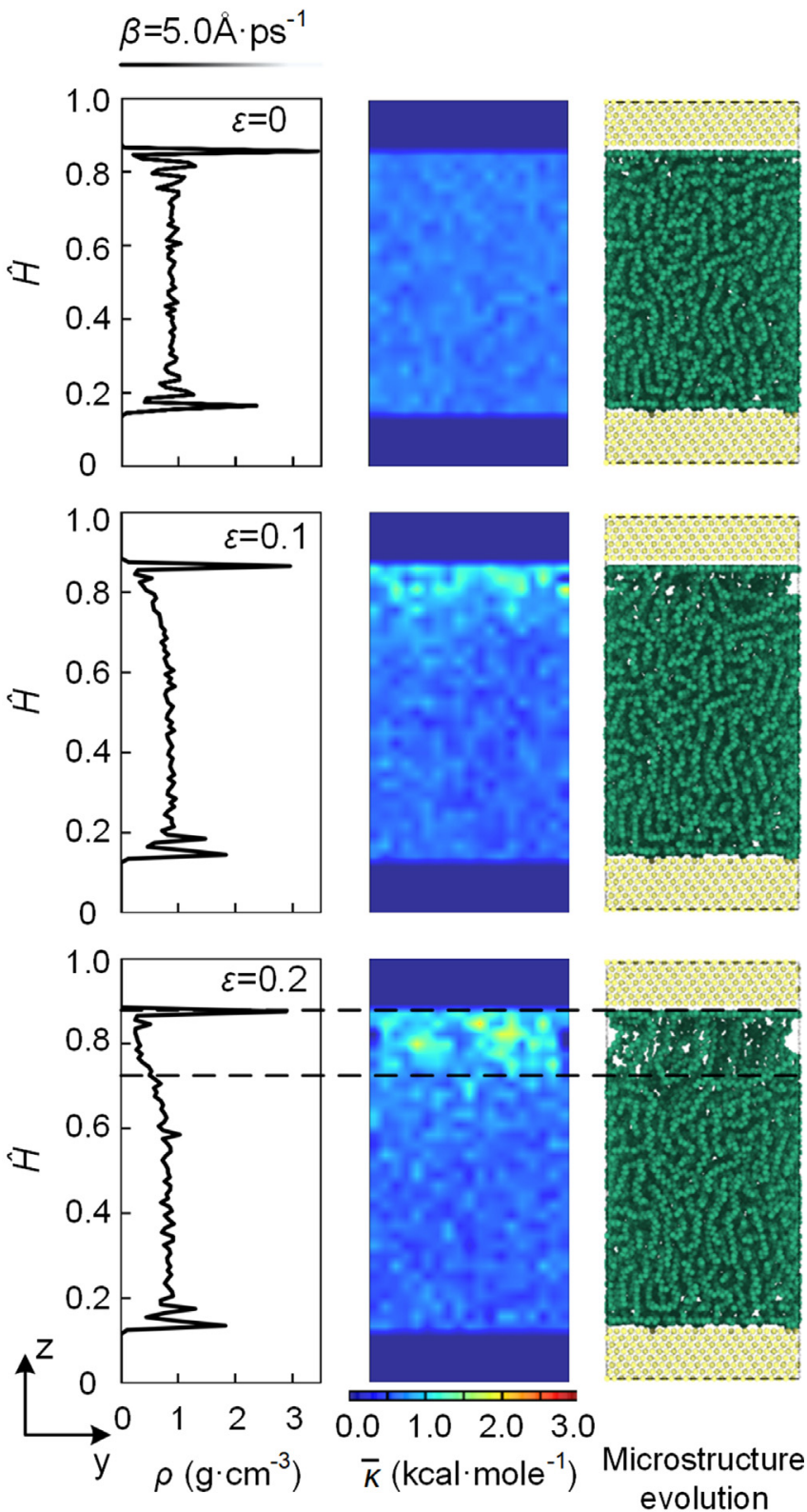


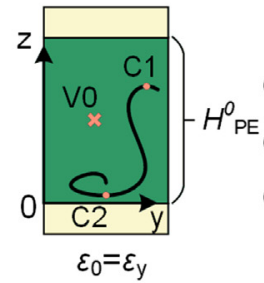

Geometry criteria:

(1) $z_{C_{2}}<z_{V_{0}}$

(2) $z_{C 1}>z_{v 0}$

(3) $Z_{C 1}-Z_{C 2} \geq 1 / 2 \cdot H_{P E}^{0}$
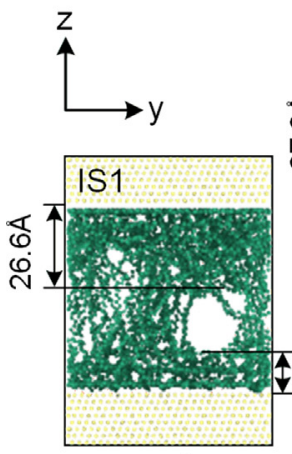

$d=39.3 \AA$
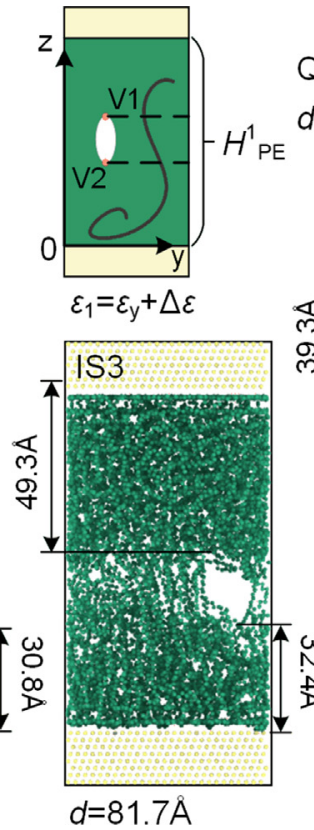

Quasi static region size:

$d=H_{\mathrm{PE}}^{1}-\mathrm{Z}_{\mathrm{V} 1}+\mathrm{Z}_{\mathrm{V} 2}$

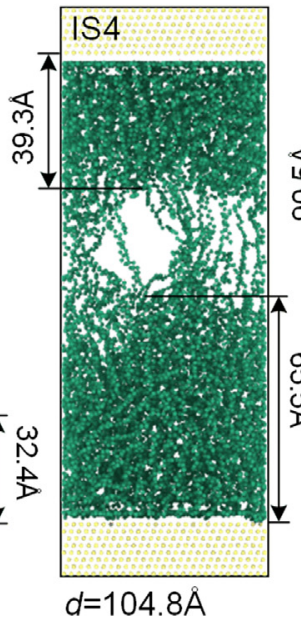

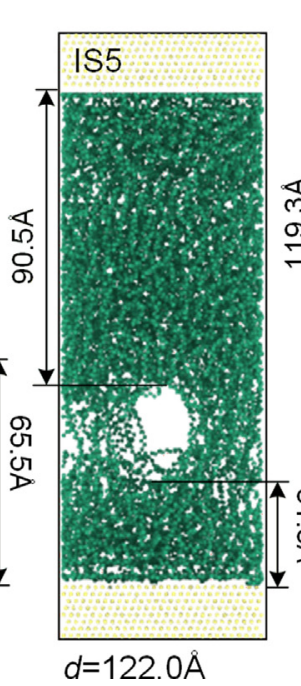

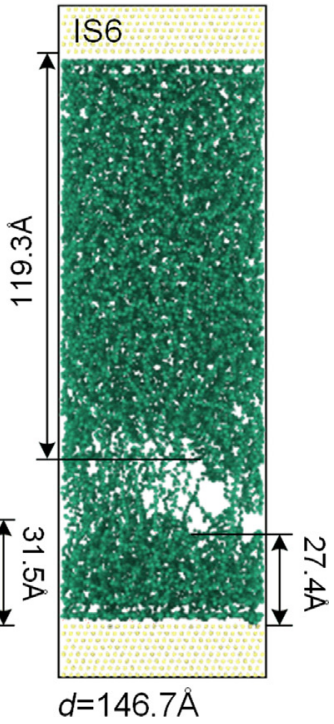

Fig. 8. Post-yielding micro-structural views of CPC sandwiches with different thicknesses of PE layer.

shown in Fig. 10(b), the degree of entanglement increases slightly in a small range and reaching a constant as $H_{\mathrm{n}}$ increases. The effect of entanglement is so subtle that can be ignored in the current study. In the contrary, the bridging effect is significant as illustrated in Fig. 10(c). It can be concluded that the thickness effect is primarily controlled by the bridging effect. Furthermore, the sensitivity of thickness effect is high when $H_{\mathrm{n}}$ is less than 2.5. As $H_{\mathrm{n}}$ increases, the thickness effect shrinks gradually.

\section{Conclusions}

In this paper, the failure behavior of CPC sandwich at microscopic scale was simulated by applying a continuous movement of copper layer with a constant velocity in MD simulation. The rationality of MD modeling was validated by the sensitivity analysis of parameters, such as mixing rules in describing the interaction between the wall $(\mathrm{Cu})$ and the sandwich layer (PE), model size, relaxation time for equilibrium and initial velocity distribution. The damage evolution of the structure during tension was illustrated through several microscale approaches such as atomic kinetic energy, atomic-stress and microstructure evolution. Finally, we obtained the main conclusions as follows.

\section{- Bridging chains \\ - High stress chains \\ Other chains}
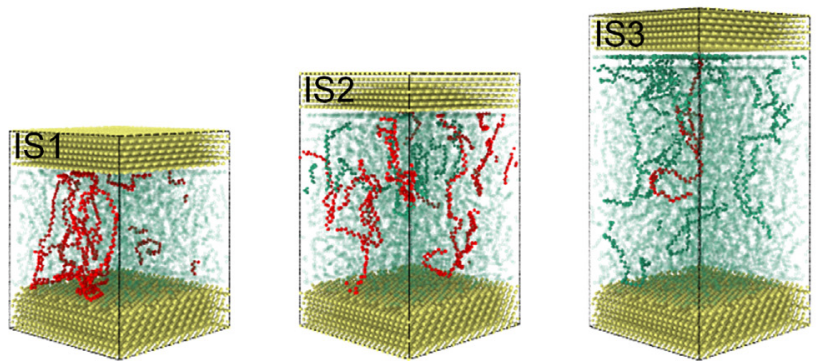

The evolution of stress-strain relation is similar but not complete corresponding to that of each component of potential energy. The peak of non-bond energy shows a delay compared to the yield point in stressstrain curve. The evolution of trans-fraction as shown in Fig. 5(c) provides an evidence to the delay phenomenon, which indicates that the instability of the structure leads to the damage initiation. Thereafter, the trans-fraction curve shows a kick, which is a phenomenon corresponding to an energy transport process during tension, resulting in the slope change of the stress-strain curve. Therefore, the dihedral distribution, which is a significant indicator of microstructure evolution, plays a crucial role in the damage of microstructure, which determines the mechanical responses of CPC.

A trough in the density profile and a local high temperature region were observed during the failure process of CPC, which occur simultaneously with the void-nucleation. Consequently, both of them are able to predict the variation of micro-void nucleation position resulted from different loading conditions.

The thickness-dependence of yield strength is co-determined by the entanglement and bridging effects of the linear chains in CPC sandwich. Compared with the negligible effect of entanglement in the current study, bridging effect has a more direct relation to the yield strength of

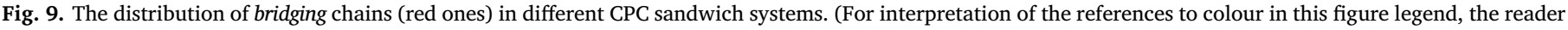
is referred to the web version of this article.) 

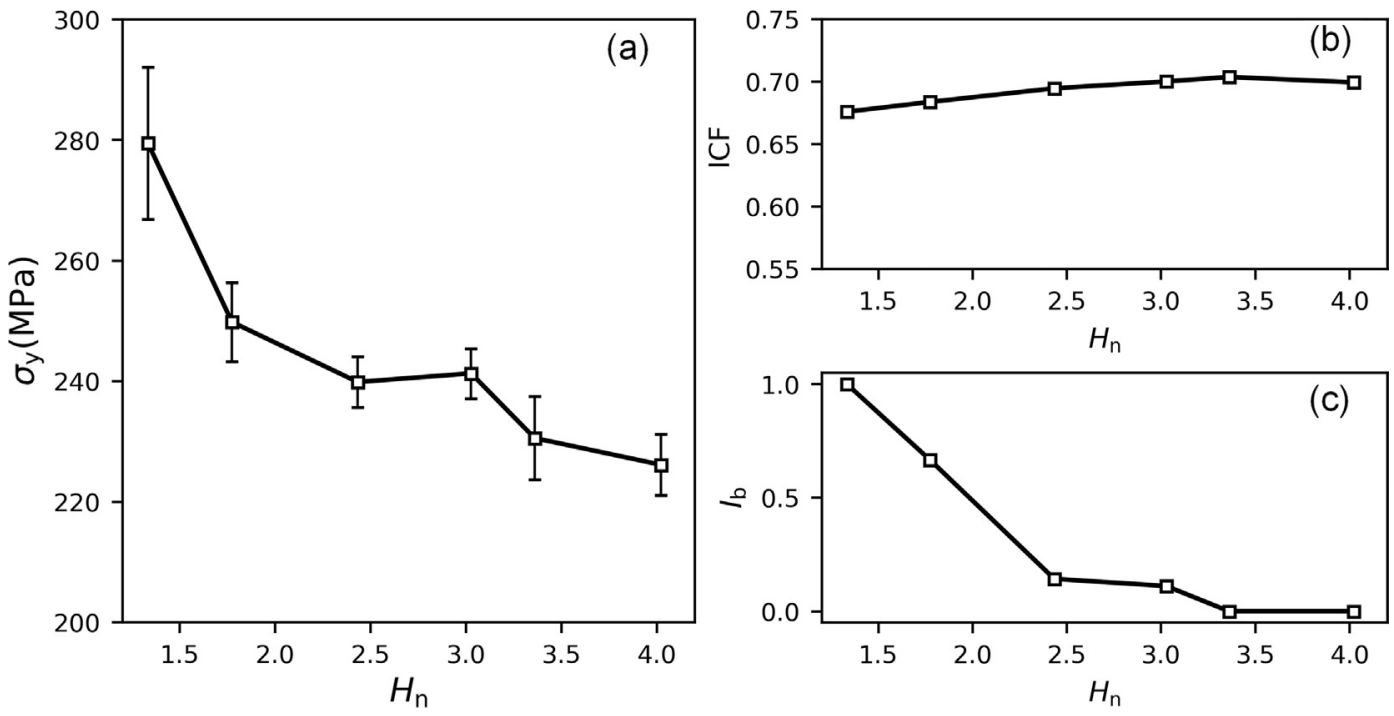

Fig. 10. The variation of (a) the yield strength $\sigma_{\mathrm{y}}$ of the statistical results of six simulations, (b) degree of entanglement of a specific sample of $H_{\mathrm{n}}$ and (c) the bridging parameter $I_{\mathrm{b}}$ with respect to the normalized thickness of the CPC interface structure of a specific sample of $H_{\mathrm{n}}$.

CPC sandwich, which is in reverse proportion with the yield strength. The smaller thickness of PE layer brings higher bridging parameter and higher yield strength. As the thickness increases, the yield strength decreases gradually to reach a stability.

\section{Data availability}

The raw/processed data required to reproduce these findings cannot be shared at this time as the data also forms part of an ongoing study.

\section{CRediT authorship contribution statement}

Changyu Meng: Methodology, Formal Analysis, Investigation, Data Curation, Writing-Original Draft, Visualization. Lijuan Liao: Conceptualization, Supervision, Funding Acquisition, Methodology, Writing- Review \& Editing. Chenguang Huang: Supervision.

\section{Acknowledgements}

This work was funded by the National Natural Science Foundation of China grant 11672314. The computations were supported by National Supercomputing Center in Shenzhen (Shenzhen Cloud Computing Center) and the Computing Facility, Institute of Mechanics, Chinese Academy of Sciences. The authors declare that there is no conflict of interest regarding the publication of this article.

\section{Appendix A. Supplementary material}

In the Section S1 of the supplemental material, the comparison of three common mixing rules was carried out to illustrate their influences on the mechanical responses for the CPC sandwich. In Section S2, three main differences on the tensile responses between the amorphous PE and CPC sandwich structures were discussed. Supplementary data associated with this article can be found, in the online version, at https:// doi.org/10.1016/j.commatsci.2018.08.011.

\section{References}

[1] A.V. Pocius, Adhesion and Adhesives Technology, third ed., Hanser Publications, Munich, 2012https://doi.org/10.3139/9783446431775.fm.

[2] K.F. Mansfield, D.N. Theodorou, Atomistic simulation of a glassy polymer/graphite interface, Macromolecules 24 (15) (1991) 4295-4309, https://doi.org/10.1021/ ma00015a011.

[3] M. Rubinstein, R.H. Colby, Polymer Physics, Oxford University Press, New York,
2003.

[4] E.J. Kramer, L.L. Berger, Fundamental processes of craze growth and fracture, in: H.H. Kausch (Ed.), Crazing in Polymers, Springer, vol. 2, Berlin, Heidelberg, 1990, pp. 1-68 https://doi.org/10.1007/BFb0018018.

[5] A. Adnan, C.T. Sun, Effect of adhesive thickness on joint strength: a molecular dynamics perspective, J. Adhes. 84 (5) (2008) 401-420, https://doi.org/10.1080/ 00218460802089239.

[6] G. Ji, Z. Ouyang, G. Li, Effects of bondline thickness on Mode-II interfacial laws of bonded laminated composite plate, Int. J. Fract. 168 (2) (2011) 197-207, https:// doi.org/10.1007/s10704-010-9571-9.

[7] G. Ji, Z. Ouyang, G. Li, S. Ibekwe, S.S. Pang, Effects of adhesive thickness on global and local Mode-I interfacial fracture of bonded joints, Int. J. Solids. Struct. 47 (18-19) (2010) 2445-2458, https://doi.org/10.1016/j.ijsolstr.2010.05.006.

[8] L.J. Liao, T. Sawa, C.G. Huang, Experimental and FEM studies on mechanical properties of single-lap adhesive joint with dissimilar adherends subjected to impact tensile loadings, Int. J. Adhes. Adhes. 44 (2013) 91-98, https://doi.org/10.1016/j. ijadhadh.2013.02.007.

[9] U. Kulmi, S. Basu, A molecular dynamics study of the failure modes of a glassy polymer confined between rigid walls, Model. Simul. Mater. Sci. Eng. 14 (6) (2006) 1071-1093, https://doi.org/10.1088/0965-0393/14/6/013.

[10] C. Yan, Y. Mai, Q. Yuan, L. Ye, J. Sun, Effects of substrate materials on fracture toughness measurement in adhesive joints, Int. J. Mech. Sci. 43 (9) (2001) 2091-2102, https://doi.org/10.1016/S0020-7403(01)00030-3.

[11] B. Xu, S. Yin, Y. Wang, H. Li, B. Zhang, R.O. Ritchie, Long-fiber reinforced thermoplastic composite lattice structures: fabrication and compressive properties, Compos. Pt. A-Appl. Sci. Manuf. 97 (2017) 41-50, https://doi.org/10.1016/j. compositesa.2017.03.002.

[12] J. Xu, X. Gao, C. Zhang, S. Yin, Flax fiber-reinforced composite lattice cores: a lowcost and recyclable approach, Mater. Des. 133 (2017) 444-454, https://doi.org/10. 1016/j.matdes.2017.07.066.

[13] S. Yin, J. Li, B. Liu, K. Meng, Y. Huan, S.R. Nutt, J. Xu, Honeytubes: hollow lattice truss reinforced honeycombs for crushing protection, Compos. Struct. 160 (2017) 1147-1154, https://doi.org/10.1016/j.compstruct.2016.11.007.

[14] L.F.M. da Silva, P.J.C. das Neves, R.D. Adams, J.K. Spelt, Analytical models of adhesively bonded joints—Part I: literature survey, Int. J. Adhes. Adhes. 29 (3) (2009) 319-330, https://doi.org/10.1016/j.ijadhadh.2008.06.005.

[15] L.F.M. da Silva, P.J.C. das Neves, R.D. Adams, A. Wang, J.K. Spelt, Analytical models of adhesively bonded joints-Part II: comparative study, Int. J. Adhes. Adhes. 29 (3) (2009) 331-341, https://doi.org/10.1016/j.ijadhadh.2008.06.007.

[16] J.L. Högberg, Mixed mode cohesive law, Int. J. Fract. 141 (3) (2006) 549-559, https://doi.org/10.1007/s10704-006-9014-9.

[17] L.J. Liao, T. Sawa, C.G. Huang, Numerical analysis on load-bearing capacity and damage of double scarf adhesive joints subjected to combined loadings of tension and bending, Int. J. Adhes. Adhes. 53 (2014) 65-71, https://doi.org/10.1016/j. ijadhadh.2014.01.010.

[18] R.D.S.G. Campilho, M.D. Banea, J.A.B.P. Neto, L.F.M.d. Silva, Modelling of singlelap joints using cohesive zone models: effect of the cohesive parameters on the output of the simulations, J. Adhes. 88 (4-6) (2012) 513-533, https://doi.org/10 1080/00218464.2012.660834.

[19] P. Martiny, F. Lani, A.J. Kinloch, T. Pardoen, A multiscale parametric study of mode I fracture in metal-to-metal low-toughness adhesive joints, Int. J. Fract. 173 (2) (2012) 105-133, https://doi.org/10.1007/s10704-011-9667-x.

[20] T. Pardoen, T. Ferracin, C.M. Landis, F. Delannay, Constraint effects in adhesive joint fracture, J. Mech. Phys. Solids 53 (9) (2005) 1951-1983, https://doi.org/10. 1016/j.jmps.2005.04.009.

[21] B.J. Alder, T.E. Wainwright, Phase transition for a hard sphere system, J. Chem. 
Phys. 27 (5) (1957) 1208-1209, https://doi.org/10.1063/1.1743957.

[22] J. Rottler, M.O. Robbins, Growth, microstructure, and failure of crazes in glassy polymers, Phys. Rev. E 68 (1) (2003) 1-18, https://doi.org/10.1103/PhysRevE.68. 011801.

[23] H. Fan, C.K. Wong, M.M. Yuen, A multiscale method to predict delamination in Cuepoxy systems in electronic packages, in: 59th Electronic Components and Technology Conference, 2009, ECTC 2009, IEEE, 2009, pp. 246-250. https://doi. org/10.1109/ECTC.2009.5074024.

[24] X.W. Zhou, N.R. Moody, R.E. Jones, J.A. Zimmerman, E.D. Reedy, Molecular-dynamics-based cohesive zone law for brittle interfacial fracture under mixed loading conditions: effects of elastic constant mismatch, Acta Mater. 57 (16) (2009) 4671-4686, https://doi.org/10.1016/j.actamat.2009.06.023.

[25] L.J. Liao, C.G. Huang, Numerical analysis of effects of adhesive type and geometry on mixed-mode failure of adhesive joint, Int. J. Adhes. Adhes. 68 (2016) 389-396, https://doi.org/10.1016/j.ijadhadh.2015.12.013.

[26] L.J. Liao, C.G. Huang, T. Sawa, Effect of adhesive thickness, adhesive type and scarf angle on the mechanical properties of scarf adhesive joints, Int. J. Solids. Struct. 50 (25-26) (2013) 4333-4340, https://doi.org/10.1016/j.ijsolstr.2013.09.005.

[27] J.E. Shepherd, Multiscale Modeling of the Deformation of Semi-Crystalline Polymers, 2006.

[28] W. Paul, D.Y. Yoon, G.D. Smith, An optimized united atom model for simulations of polymethylene melts, J. Chem. Phys. 103 (4) (1995) 1702-1709, https://doi.org/ 10.1063/1.469740.

[29] S. Plimpton, Fast parallel algorithms for short-range molecular dynamics, J. Comput. Chem. 117 (1) (1995) 1-19, https://doi.org/10.1006/jcph.1995.1039.

[30] S. Alexander, Visualization and analysis of atomistic simulation data with OVITO-the Open Visualization Tool, Modelling Simul. Mater. Sci. Eng. 18 (1) (2010) 015012, , https://doi.org/10.1088/0965-0393/18/1/015012.

[31] S.L. Mayo, B.D. Olafson, W.A. Goddard, DREIDING: a generic force field for molecular simulations, J. Phys. Chem. 94 (26) (1990) 8897-8909, https://doi.org/10. 1021/j100389a010.
[32] D. Hossain, M.A. Tschopp, D.K. Ward, J.L. Bouvard, P. Wang, M.F. Horstemeyer, Molecular dynamics simulations of deformation mechanisms of amorphous polyethylene, Polymer 51 (25) (2010) 6071-6083, https://doi.org/10.1016/j.polymer. 2010.10.009.

[33] H. Heinz, R.A. Vaia, B.L. Farmer, R.R. Naik, Accurate simulation of surfaces and interfaces of face-centered cubic metals using 12-6 and 9-6 Lennard-Jones potentials, J. Phys. Chem. C. 112 (44) (2008) 17281-17290, https://doi.org/10.1021/ jp801931d.

[34] J. Han, R.L. Jaffe, D.Y. Yoon, Conformational characteristics of polymethylene chains in melts and in various phantom chains from explicit atom molecular dynamics simulations, Macromolecules 30 (23) (1997) 7245-7252, https://doi.org/ 10.1021/ma961772b.

[35] L. Martínez, R. Andrade, E.G. Birgin, J.M. Martínez, PACKMOL: a package for building initial configurations for molecular dynamics simulations, J. Comput. Chem. 30 (13) (2009) 2157-2164, https://doi.org/10.1002/jcc.21224.

[36] S.J. Nikkhah, M.R. Moghbeli, S.M. Hashemianzadeh, Dynamic study of deformation and adhesion of an amorphous polyethylene/graphene interface: a simulation study, Macromol. Theor. Simul. 25 (6) (2016) 533-549, https://doi.org/10.1002/ mats.201600069.

[37] J.L. Jordan, D.T. Casem, J.M. Bradley, A.K. Dwivedi, E.N. Brown, C.W. Jordan, Mechanical properties of low density polyethylene, J. Dyn. Behav. Mater. 2 (4) (2016) 411-420, https://doi.org/10.1007/s40870-016-0076-0.

[38] J. Delhommelle, P. Millie, Inadequacy of the Lorentz-Berthelot combining rules for accurate predictions of equilibrium properties by molecular simulation, Mol. Phys. 99 (8) (2001) 619-625, https://doi.org/10.1080/00268970010020041.

[39] W. Krauth, Statistical Mechanics: Algorithms and Computations, Oxford University Press, Oxford, 2006.

[40] X. Yu, B. Kong, X. Yang, Molecular dynamics study on the crystallization of a cluster of polymer chains depending on the initial entanglement structure, Macromolecules 41 (18) (2008) 6733-6740, https://doi.org/10.1021/ma800172t. 